\title{
TWIST-ROLL SPUN KNOTS
}

\author{
MASAKAZU TERAGAITO
}

(Communicated by Ronald Stern)

\begin{abstract}
We study 2-knots obtained from 1-knots by a combination of twistspinning and roll-spinning and ask whether they are nontrivial. It is proved that, under a certain assumption, the resulting 2-knot is always nontrivial when the 1-knot is not a torus knot, making use of the Cyclic Surgery Theorem.
\end{abstract}

Let $K$ be a knot in $S^{3}$, and let $\tau^{m} \rho^{n}(K)$ denote the $(m, n)$ twist-roll spun 2-knot in $S^{4}$ (see [L]). Zeeman [Z] showed that if $m \neq 0$ then $\tau^{m}(K)$ is a fibered 2-knot with closed fiber the $m$-fold cyclic branched covering space of $S^{3}$ branched over $K$. In particular, $\tau^{ \pm 1}(K)$ is always trivial. However, for a nontrivial knot $K, \tau^{m}(K)$ is nontrivial if $|m| \neq 1$ by the generalized Smith conjecture [MB]. In [T] it is shown that $\rho^{n}(K)$ of nontrivial $K$ is nontrivial for any $n$. One of the results for general $m, n$ is that if $K$ is the $(p, q)$ torus knot then $\tau^{m} \rho^{n}(K)$ is equivalent to $\tau^{m-n p q}(K)$ [L, Corollary 6.4]. Hence it is nontrivial if and only if $m-n p q \neq \pm 1$.

We treat the case that $K$ is not a torus knot.

Theorem 1. Suppose that $K$ is not a torus knot. Then $\tau^{m} \rho^{n}(K)$ is nontrivial if $|n| \geq 2$.

Proof. It is easy to see that $\tau^{m} \rho^{n}(K)$ is equivalent to $\tau^{-m} \rho^{-n}(K)$, so we may assume that $m \geq 0$. If $m=0, \rho^{n}(K)$ is nontrivial [T, Theorem 2]. We consider $m>0$. By Litherland [L, Corollary 5.2], $\tau^{m} \rho^{n}(K)$ is a fibered knot with closed fiber $E_{m}(1 / n) . E_{m}(1 / n)$ denotes the closed 3-manifold obtained from the $m$-fold cyclic covering space $E_{m}$ of the knot exterior $E$ by $(1 / n)$ Dehn filling. (We parametrize the slopes on $\partial E_{m}$ with respect to the induced framing from $\partial E$.) In case $m=1$, we denote $E_{1}(s)$ by $K(s)$ after [CGLS]. Note that the canonical covering transformation $g$ of $E_{m}$ extends to a periodic automorphism of $E_{m}(s)$ for any slope $s$ on $\partial E_{m}$, which may fix pointwise the core of the attached solid torus.

The commutator subgroup of $\pi_{1}\left(S^{4}-\tau^{m} \rho^{n}(K)\right)$ is isomorphic to $\pi_{1} E_{m}(1 / n)$. If $m=1$, then $E_{1}(1 / n)$ is just $K(1 / n)$. Then, by [CGLS, Corollary 2], $K(1 / n)$ is not simply-connected if $|n| \geq 2$. Therefore $\tau^{1} \rho^{n}(K)$ is nontrivial. We assume that $m>1$. Now suppose that $\tau^{m} \rho^{n}(K)$ is trivial. Then $E_{m}(1 / n)$ is

Received by the editors January 5, 1993.

1991 Mathematics Subject Classification. Primary 57Q45. 
simply-connected. Let $\hat{g}$ denote the extension to $E_{m}(1 / n)$ of the canonical covering transformation $g$ of $E_{m}$. Let $\operatorname{Fix}\left(\hat{g}^{i}\right)$ be the fixed-point set of $\hat{g}^{i}$.

We distinguish two cases.

Case 1. $\operatorname{Fix}\left(\hat{g}^{i}\right)=\varnothing \quad(1 \leq i \leq m-1)$.

In this case $\hat{g}$ generates a free $Z_{m}$-action on $E_{m}(1 / n)$ with quotient $K(s)$ for some slope $s$ on $\partial E$. In fact, it is not hard to see that $s=m / n$. (In the present setting, we have $(m, n)=1$, and so $m / n$ surely determines a slope.) Since the quotient map $E_{m}(1 / n) \rightarrow K(m / n)$ is an $m$-fold cyclic covering, we have

$$
\pi_{1} K(m / n) \cong Z_{m}
$$

But this contradicts the fact that if $K$ is not a torus knot, $K(m / n)$ does not have cyclic fundamental group unless $n=0, \pm 1$ [CGLS, Corollary 1].

Case 2. $\operatorname{Fix}\left(\hat{g}^{i}\right) \neq \varnothing$ for some $i \quad(1 \leq i \leq m-1)$.

In this case $\operatorname{Fix}\left(\hat{g}^{i}\right)$ is the core $C$ of the attached solid torus $V$. Then, by the generalized Smith conjecture [MB], $C$ bounds a disk in $E_{m}(1 / n)$. Therefore $\partial E_{m}$ is compressible in $E_{m}$, and hence $E_{m}$ is a solid torus (since $E_{m}$ is irreducible). This implies that $K$ is trivial, a contradiction.

Theorem 2. Suppose that $K$ is a nontrivial amphicheiral knot. Then $\tau^{m} \rho^{n}(K)$ is trivial if and only if $m= \pm 1$ and $n=0$.

Proof. Recall that nontrivial torus knots are not amphicheiral. The result follows from Theorem 1 and the fact that for a nontrivial amphicheiral knot $K$, $K(m)$ does not have cyclic fundamental group for any $m \neq 0$ [CGLS, Corollary 4].

Theorem 3. Suppose that $K$ is a composite knot. Then $\tau^{m} \rho^{n}(K)$ is trivial if and only if $m= \pm 1$ and $n=0$.

Proof. This follows from Theorem 1 and the fact that nontrivial Dehn surgery on a composite knot does not yield a manifold with cyclic fundamental group (see [Go, Lemma 7.1]).

In view of Theorem $1, \tau^{m} \rho^{ \pm 1}(K)$ may be trivial for a nontorus knot $K$. But it follows from [CGLS, Corollary 1] that $\tau^{m} \rho^{1}(K)$ and $\tau^{m} \rho^{-1}(K)$ cannot both be trivial. We give examples of a knot $K$ such that $\tau^{m} \rho(K)$ is trivial. There are many examples of knots such that some Dehn surgeries on them yield lens spaces [BR, FS, Be, Ga]. Such knots yield trivial twist-roll spun knots as well.

Theorem 4. Let $K$ be a knot in $S^{3}$. If $m$-Dehn surgery on $K$ yields a lens space, then $\tau^{m} \rho(K)$ is trivial.

Proof. We may assume that $m>0$. Recall that $\tau^{m} \rho(K)$ is a fibered knot with closed fiber $E_{m}(1)$. As in the proof of Theorem $1, E_{m}(1)$ is an $m$-fold covering space of $K(m)$, which is a lens space with fundamental group $Z_{m}$. Hence $E_{m}(1)$ is the universal covering space of $K(m)$, and so it must be $S^{3}$. This implies that $\tau^{m} \rho(K)$ is trivial.

For instance, 18- and 19-Dehn surgeries on the $(-2,3,7)$ pretzel knot $K$ yield lens spaces [FS]. Thus $\tau^{18} \rho(K)$ and $\tau^{19} \rho(K)$ are trivial.

Conversely, if $\tau^{m} \rho(K)$ is trivial for some $m$, then $E_{m}(1)$ is simplyconnected, and hence $K(m)$ has cyclic fundamental group. But it is unknown that $K(m)$ is a lens space in general. 


\section{REFERENCES}

[BR] J. Bailey and D. Rolfsen, An unexpected surgery construction of a lens space, Pacific J. Math. 71 (1977), 295-298.

[Be] J. Berge, The knots in $D^{2} \times S^{1}$ which have nontrivial Dehn surgeries that yield $D^{2} \times S^{1}$, Topology Appl. 38 (1991), 1-19.

[CGLS] M. Culler, C. McA. Gordon, J. Luecke, and P. B. Shalen, Dehn surgery on knots, Ann. of Math. (2) 125 (1987), 237-300.

[FS] R. Fintushel and R. Stern, Constructing lens spaces by surgery on knots, Math. Z. 175 (1980), 33-51.

[Ga] D. Gabai, Surgery on knots in solid tori, Topology 28 (1989), 1-6.

[Go] C. Gordon, Dehn surgery and satellite knots, Trans. Amer. Math. Soc. 275 (1983), 687-708.

[L] R. A. Litherland, Deforming twist-spun knots, Trans. Amer. Math. Soc. 250 (1979), 311-331.

[MB] J. Morgan and H. Bass, The Smith conjecture, Pure and Appl. Math., Academic Press, London and New York, 1984.

[T] M. Teragaito, Roll-spun knots, Proc. Cambridge Philos. Soc. 113 (1993), 91-96.

[Z] E. C. Zeeman, Twisting spun knots, Trans. Amer. Math. Soc. 115 (1965), 471-495.

Department of Mathematics, Kobe University, Rokko 1-1, Nada, Kobe 657, Japan 